\title{
The Central Kiloparsec-Scale Structure of Galaxies
}

\author{
Roeland P. van der Marel \\ Space Telescope Science Institute, 3700 San Martin Drive, Baltimore, \\ $M D$ 21218, USA
}

\begin{abstract}
This review summarizes some aspects of the central kiloparsec scale structure of galaxies, and in particular spiral galaxies, elliptical galaxies and merger remnants. The focus is on results from optical and near-IR imaging and spectroscopy, with emphasis on recent work with the Hubble Space Telescope.
\end{abstract}

\section{Introduction}

This paper provides an overview of some aspects of the structure of galaxies in the central kiloparsec, as relevant in the context of the symposium topic: 'Galaxies and Their Constituents at the Highest Angular Resolutions'.

For the purpose of this introduction, let us consider high angular resolution to mean $\sim 0.1$ arcsec or better. This angular scale corresponds to a linear scale of $1 \mathrm{pc}$ at a distance of $2 \mathrm{Mpc}$. While many important topics can be studied on the parsec scale in galaxy centers (see other reviews in this volume), such studies are limited to only a small fraction of the observational tools and astronomical targets available to astronomers. In particular, one must either: (a) look at very nearby galaxies (e.g., in the Local Group); (b) use the limited set of observational techniques that provide resolutions $\ll 0.1 \operatorname{arcsec}$ (e.g., very long baseline radio interferometry); or (c) study the global properties of regions that are not spatially resolved (e.g., AGN broad-line regions of accretion disks).

By contrast, structures in the central kiloparsec of galaxies are easily accessible except for very distant targets. For 0.1 arcsec to correspond to $1 \mathrm{kpc}$ one must go to a distances of $2 \mathrm{Gpc}$, corresponding to a redshift $z \approx 0.5$. Hence, a complete review of the central kiloparsec scale structure of galaxies would necessarily have to encompass a discussion of the full range of observational techniques (imaging, spectroscopy, polarimetry, interferometry, etc.) and wavelength ranges (radio, sub-mm/mm, far/mid-IR, near-IR/optical, UV, Xray, $\gamma$-ray) available, for the different structural and physical components (stars, gas, dust, black holes, dark matter, etc.) that are present in the large variety of interesting galaxy types in the Universe (various Hubble types, galaxy mergers and interactions, starburst galaxies, ultra-luminous IR galaxies, dwarf galaxies, low-surface brightness galaxies, LINERS, Seyfert galaxies, radio galaxies, quasars, BL Lac objects, blazars, etc.). The main challenge is then to put the vast amounts of observational information together into one coherent picture of galaxy structure and evolution. However, such a detailed discussion is far beyond what is feasible in the context of a brief review. So instead I focus here on 

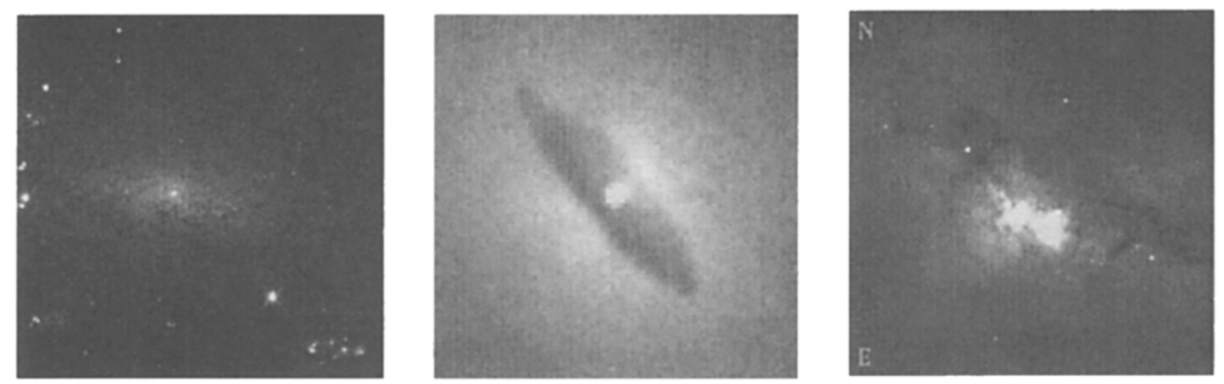

Figure 1. (a) HST/WFPC2 I-band image of NGC 6509, a late-type spiral galaxy with a nuclear star cluster (see Section 2.2; Böker et al. 2001b). (b) HST/WFPC2 $V$-band image of NGC 315, an elliptical galaxy with a nuclear dust disk (see Section 3.2; Verdoes Kleijn et al. 1999). (c) HST/WFPC2 I-band image of NGC 6240, a ULIRG and merger remnant with an obscured AGN (see Section 4.1; van der Marel et al. 2001).

a small sub-topic, namely results from imaging and spectroscopy in the optical and near-IR wavelength regimes, in particular from recent work with the Hubble Space Telescope (HST). I describe some new insights into the central kiloparsec scale structure of spiral galaxies, elliptical galaxies and merger remnants.

\section{Spiral Galaxies}

\subsection{Bulges}

The central regions of spiral galaxies have traditionally been well studied at kiloparsec scales using ground-based observations. Results from such studies have shown that spiral galaxies have bulges, and these bulges diminish progressively in prominence from early-type spirals to late-type spirals. This progression forms one of the bases of the Hubble sequence classification. The majority of earlytype spirals ( $\mathrm{S} 0 \mathrm{a}-\mathrm{Sab}$ ) have bulges with brightness profiles that follow an $R^{1 / 4}$ law. Bulges of this kind have traditionally been viewed as 'small ellipticals' (e.g., Bender, Burstein \& Faber 1992). By contrast, most intermediate (Sb-Sc) type spirals have bulges that follow an exponential profile, and are of lower surface brightness and density than their $R^{1 / 4}$ counterparts. In late type spirals (Scd and later type) virtually all bulges are of this exponential type (e.g. Andredakis et al. 1995; Courteau, de Jong \& Broeils 1996). The physical processes that govern the formation of the different types of bulges remain a topic of debate; see, e.g., the review by Wyse, Gilmore \& Franx (1997) and the proceedings of a recent STScI conference (Carollo, Ferguson \& Wyse 1999).

\subsection{Nuclear Star Clusters}

Recent HST observations at both optical and near-IR wavelengths (e.g., Carollo, Stiavelli \& Mack 1998; Böker et al. 1999a) have revealed that a compact, 
photometrically distinct star cluster is often present in the dynamical center of spiral galaxies of all Hubble types (an example is shown in Fig. 1a).

To understand the origin and nature of these star clusters it is desirable to know their ages. One possible approach is to attempt to measure the stellar velocity dispersion of the cluster from high spectral resolution ground-based absorption line spectra. We have succeeded in doing this from the ground (with IRTF) for one very nearby galaxy, IC $342(D \approx 2 \mathrm{Mpc})$. This yields the mass of the cluster $\left(\sim 6 \times 10^{6} M_{\odot}\right)$, and hence the $M / L$ from comparison with the observed luminosity. Stellar population synthesis models for the observed $M / L$ then yield the cluster age. For IC 342 we find an age of $10^{6.8-7.8}$ years (Böker et al. 1999b). Unfortunately, most of the observed star clusters are much fainter than that in IC 342, making this approach extremely challenging. A better approach may therefore be to construct population synthesis models for low resolution spectra, which are easier to obtain at adequate $\mathrm{S} / \mathrm{N}$. We recently demonstrated the feasibility of this approach for the nearby starburst galaxy NGC 4449 (Böker et al. 2001a). A ground-based optical echellette spectrum shows strong Balmer absorption lines and a pronounced $4000 \AA$ break, both reddening-free indicators of the strength of a young population. Data-model comparison shows that the light of the cluster is dominated by a very young (6-10 Myr) population of stars. This conclusion is supported by an independent analysis of broad-band colors and a near-IR spectrum (Gelatt, Hunter \& Gallagher 2001). Interestingly, many of the nuclear star clusters that have so far been studied in detail turn out to be young. In addition to the two galaxies already mentioned, our own Milky Way has a central cluster that is only $\sim 10^{6.5}$ years old (Krabbe et al. 1995; Najarro et al. 1997), and M31 and M33 have blue nuclei when viewed at HST resolution that are quite possibly young star clusters (Lauer et al. 1998). However, it should be kept in mind that the existing studies have probably been biased towards the brightest, and hence youngest, clusters. A complete unbiased study is therefore called for. Such a study will require the superior spatial resolution of HST, to cleanly separate the cluster light (with a half-light radius of $\sim 0.1$ arcsec) from that of the underlying disk.

\subsection{Bars}

Bars are very common in spiral galaxies. Estimates from the RC3 suggest that $50-60 \%$ of spiral galaxies are barred. More detailed analysis of near-IR images suggests that the fraction of barred galaxies may be as high as $60-80 \%$ (e.g., Knapen, Shlosman \& Peletier 2000). The Milky Way is now known to be barred as well (e.g., Kuijken 1995; Englmaier \& Gerhard 1999). Bars have the potential of causing secular evolution in galaxy disks, and may be responsible for the formation of bulges. Numerical simulations have shown that dynamical resonances in the non-axisymmetric potential of a stellar bar provide an efficient mechanism for the dissipation of angular momentum and the subsequent infall of disk gas towards the galaxy center (e.g. Athanassoula 1992a,b). Of particular interest in this context is a model suggested by Friedli \& Benz (1993) and developed further by Norman, Sellwood \& Hasan (1996). They pointed out that build-up of a central mass concentration can dissolve stellar bars, and lead to the formation of a bulge via collective bending instabilities (Raha et al. 1991; Merritt \& Sellwood 1994). The Norman et al. simulations show that only $5 \%$ of the combined disk 
and bar mass in a central concentration is sufficient to destroy a bar on very short timescales, leading to a bulge-like distribution of stars. Both a central star cluster and/or a central black hole (now believed to be ubiquitous in galaxies; e.g., Kormendy \& Richstone 1995; Magorrian et al. 1998; van der Marel 1999; Ferrarese \& Merritt 2000; Gebhardt et al. 2000) could therefore potentially play a role in the destruction of bars. Accurate statistics of nuclear star cluster and black hole masses in spiral galaxies are required to address this quantitatively. Note also that since stellar bars are an effective funneling mechanism of gaseous matter into the central regions of galaxies, the destruction of a stellar bar may eliminate further matter infall towards the galaxy center. This may have implications both for the feeding of an AGN, and for the continuing availability of gas for the formation of new young star clusters.

\section{Elliptical Galaxies}

\subsection{Surface Brightness Cusps}

The high spatial resolution of HST has allowed astronomers to study the photometric structure of elliptical galaxy nuclei with unprecedented detail. The main result from a large number of different studies (e.g., Lauer et al. 1995; Carollo et al. 1997) has been that at the $\sim 0.1^{\prime \prime}$ resolution limit of HST, virtually all galaxies have power-law surface brightness cusps, $I \propto r^{-\gamma}$, with $\gamma>0$ and no observed transition to a homogeneous core. In addition, the surface brightness profiles fall in two categories: 'core' profiles, which have a break at a resolved radius and a shallow slope inside that radius, and 'power-law' profiles, which have a steep slope down to the resolution limit and no clear break (Faber et al. 1997). Core galaxies are defined to have $\gamma \leq 0.3$ at the HST resolution limit, while power-law galaxies generally have $\gamma \geq 0.5$. The classification in two groups is motivated by the fact that there appears to be a disjunct, rather than a continuous division in profile properties, and by the fact that the overall physical properties of core galaxies and power-law galaxies are different. Among other things, the nuclear properties correlate with luminosity. Galaxies with $M_{V}<-22$ have core profiles, galaxies with $M_{V}>-20.5$ have power-law profiles, and both profile types occur in galaxies with $-22<M_{V}<-20.5$.

The physics underlying the observed cusp slopes and correlations remains somewhat of a mystery. One possible interpretation is to assume that the cusps are due to the presence of black holes. It has long been know that the presence of a $\mathrm{BH}$ in the center of a stellar system induces a power-law cusp in the mass distribution (e.g., Bahcall \& Wolf 1976; Young 1980). In fact, the adiabatic black hole growth models of Young fit the observed cusp slopes quite well for reasonable black hole masses (van der Marel 1999). However, this may be somewhat fortuitous, given that the adiabatic black hole growth models appear oversimplified. First, they assume that the black hole grows slowly in a preexisting stellar system. Models in which black hole and galaxy formation occur simultaneously may be more realistic (e.g., Haehnelt, Natarajan \& Rees 1998). Second, they assume that galaxies initially form with homogeneous cores. There is evidence that the formation of low-luminosity ellipticals involves considerable dissipation (Bender, Burstein \& Faber 1992), and this may have caused these galaxies to have a central cusp to begin with. And third, galaxies are not ex- 
pected to evolve in isolation, but may experience mergers and accretion events. If both galaxies in a merger contain a central black hole, then the evolution of the black hole binary will lower the cusp slope near the center (Quinlan \& Hernquist 1997). It has been suggested that this is the mechanism responsible for the small cusp slopes in high-luminosity galaxies (Faber et al. 1997). However, it is not clear why this same mechanism wouldn't also lead to small cusp slopes in the denser low-luminosity galaxies. So while considerable insight has been gained in the central stellar density distribution of elliptical galaxies, much remains to be understood about the underlying physics.

\subsection{Nuclear Disks}

With HST it has been possible to study the dust and ionized gas in early-type galaxies at $\sim 10$ times better resolution than before. Interestingly, this has revealed the existence of previously unknown structures: 'nuclear disks', with typical sizes of $0.1-1 \mathrm{kpc}$. In some cases the disks are seen only in ionized gas emission (e.g., M87; Ford et al. 1994), while in other cases they have strong associated dust absorption, visible as a dust lane across the nucleus (e.g., NGC 7052; van der Marel \& van den Bosch 1998; see also Fig. 1b.). The observed morphology and kinematics of the nuclear disks imply approximately circular motion under the influence of gravity, and they have therefore been used successfully to measure the masses of central black holes. The nuclear disks are sometimes kinematically decoupled from the stellar body. In NGC 4261 the stars rotate around the major axis (indicating a prolate geometry), while the nuclear dust and gas disk rotates around the minor axis (Ferrarese, Ford \& Jaffe 1996). This leads to a picture in which gas and dust have an external origin, due to some accretion or merger event, and have settled at small radii in one of the symmetry planes of the galactic potential. HST observations show nuclear dust in $\sim 80 \%$ of early-type galaxies (van Dokkum \& Franx 1995). The dust morphology is often patchy, and not always in the form of a disk. The detection rate of nuclear dust in radio-loud systems is roughly twice that in radio-quiet systems. This indicates a possible relation between the dust and $\mathrm{BH}$ fueling.

Small stellar disks are also sometimes found, examples being the nearly edge-on disks in NGC 4342, NGC 4570 (van den Bosch, Jaffe \& van der Marel 1998) and NGC 3115 (Kormendy et al. 1996). A plausible formation scenario for these disks is the dissipative infall of gas, which settles into a disk and forms stars (Hernquist \& Barnes 1991). In such a scenario kinematical decoupling (e.g., Mehlert et al. 1997) arises naturally, because the gas maintains its own angular momentum. On the other hand, strong color and stellar population differences between the kinematically decoupled core and the main body are the exception rather than the rule (Carollo et al. 1997), indicating that the infall may have occurred at early times.

\section{Galaxy Mergers and Merger Remnants}

\subsection{Starbursts and ULIRGs}

Numerical simulations show that torques and shocks during a merger efficiently remove angular momentum from the gas in the merging galaxies, which there- 
fore flows to the galaxy center(s) (e.g., Mihos \& Hernquist 1996). The gas gets compressed, and is thus expected to form stars. This is generally confirmed observationally; e.g., HST observations of the well-known Antennae galaxies beautifully reveal wide-spread star and star cluster formation (Whitmore et al. 1999). Often the star formation is dust-enshrouded, in which case the majority of the energy is emitted in the IR. In the most spectacular cases this leads to UltraLuminous Infra-Red Galaxies (ULIRGs). Such galaxies are almost always seen to be interacting systems or merger remnants, and HST observations have shown that in $\sim 20 \%$ of ULIRGs more than two galaxies appear to be involved in the interaction (Borne et al. 2000). The gas flow towards the center in an interacting system may also fuel an AGN, which can also contribute to the observed IR emission. In some cases the presence of an (obscured) AGN is unambiguous from the observation of strong X-ray emission, as in e.g., NGC 6240 (e.g., Vignati et al. 1999; an HST image is shown in Fig. 1c). Genzel et al. (1998) have used IR emission-line diagnostic ratios from ISO data to address the nature of the energy source of ULIRGs more quantitatively. They find that $70-80 \%$ of the ULIRGs in their sample are predominantly powered by recently formed massive stars, and $20-30 \%$ by a central AGN. At least half of the sources probably have both an active nucleus and starburst activity in a 1-2 kpc diameter circumnuclear disk/ring.

\subsection{Formation of ellipticals from mergers}

The possibility that many elliptical galaxies formed from mergers of disk galaxies is a topic of continuing interest. That mergers form elliptical-like remnants has been demonstrated through numerical simulations (e.g., Hernquist 1992), and ground-based imaging has shown that many merger remnants have $\mathrm{r}^{1 / 4}$ luminosity profiles (e.g., Stanford \& Bushouse 1991). These arguments, along with the detection of shells, ripples, dust disks and kinematically decoupled cores in elliptical galaxies, support this 'merger hypothesis' (e.g., Kennicutt, Schweizer \& Barnes 1998).

Theoretical arguments indicate that it is in the nuclei of remnants where the merger hypothesis may face its most stringent test. If dynamical relaxation is the dominant physical process in mergers, then remnant nuclei will be very diffuse with large cores (Hernquist 1992), unless the progenitor nuclei were dense to begin with. If both merging galaxies contain a central black hole, then the stellar density of the merger remnant will be lower than that of the progenitor galaxies (Quinlan \& Hernquist 1997). Alternatively, if mergers are accompanied by strong gaseous dissipation and central starbursts, then the remnant may have a high stellar density and steep luminosity profile (Mihos \& Hernquist 1994).

A comparison between the observed nuclear properties of merger remnants and elliptical galaxies can shed more light on the viability of the merger hypothesis and on the physical processes that govern the structure of merger remnants. We have undertaken a study with HST/NICMOS to address this issue. Preliminary results were presented in van der Marel \& Zurek (2000). Fourteen late-stage merger remnants (identified morphologically) were imaged in $J, H$ and $K$, and for each galaxy the surface brightness profile was inferred from the data. The profiles generally show a central cusp, as do elliptical galaxies. Most of the galaxies in the sample have cusp slopes that are similar to those typical 
for ellipticals of the same luminosity. On the other hand, three of the fourteen galaxies stand out by having a higher central luminosity density than typical for elliptical galaxies. In two of these galaxies the light becomes bluer towards the center, presumably due to recent star formation. It is therefore plausible to attribute the excess luminosity density to stars formed recently from gas that has fallen towards the center, as predicted in the models of Mihos \& Hernquist (1994). The young stars will fade with time, so that these galaxies will be more similar to elliptical galaxies after another few Gyrs. So these results are not inconsistent with the hypothesis that merging disk galaxies form ellipticals. However, what fraction of ellipticals in the Universe formed from a major merger remains an open question.

It is a pleasure to thank Torsten Böker for assistance with the text of Section 2. Several of the HST projects discussed in this review are supported by grants awarded by the Space Telescope Science Institute which is operated by the Association of Universities for Research in Astronomy, Incorporated, under NASA contract NAS5-26555.

\section{References}

Andredakis, Y. C., Peletier, R. F., \& Balcells, M. 1995, MNRAS, 275, 874

Athanassoula, E. 1992a, MNRAS, 259, 328

Athanassoula, E. 1992b, MNRAS, 259, 345

Bahcall, J. N., \& Wolf R. A. 1976, ApJ, 209, 214

Bender, R., Burstein, D., \& Faber, S. M. 1992, ApJ, 399, 462

Böker, T., et al. 1999a, ApJS, 124, 95

Böker, T., van der Marel, R. P., \& Vacca, W. D. 1999b, AJ, 118, 831

Böker, T., van der Marel, R. P., Mazzuca, L., Rix, H.-W., Rudnick, G., Ho, L., \& Shields, J. 2001a, AJ, in press [astro-ph/0010542]

Böker, T., van der Marel, R. P., Laine, S., Rix, H.-W., Ho, L., \& Shields, J. $2001 \mathrm{~b}$, in preparation

Borne, K. D., Bushouse, H., Lucas, R. A., \& Colina, L. 2000, ApJ, 529, L77

Carollo, C. M., Franx, M., Illingworth, G. D., \& Forbes D. 1997, ApJ, 481, 710

Carollo, C. M., Stiavelli, M., \& Mack, J. 1998, AJ, 116, 68

Carollo, C. M., Ferguson, H. C., \& Wyse, R. F. G. 1999, 'The Formation of Galactic Bulges' (Cambridge: Cambridge University Press)

Courteau, S., de Jong, R., \& Broeils, A. 1996, ApJL, 457, L73

Englmaier, P., \& Gerhard, O. E. 1999, MNRAS, 304, 512

Faber, S. M., et al. 1997, AJ, 114, 1771

Ferrarese, L., Ford, H. C., Jaffe, W. 1996, ApJ, 470, 444

Ferrarese, L., \& Merritt, D. 2000, ApJ, 539, L9

Ford H. C., et al. 1994, ApJ, 435, L27

Friedli, D. \& Benz, W. 1993, A\&A, 268, 65

Gebhardt, K. et al. 2000, ApJ, 539, L13 
Gelatt, A. E., Hunter, D. A., \& Gallagher, J. S. 2001, PASP, in press [astro$\mathrm{ph} / 0010515]$

Genzel, R., et al. 1998, ApJ, 498, 579

Haehnelt, M. G., Natarajan, P., \& Rees, M. J. 1998, MNRAS, 300, 817

Hernquist, L., \& Barnes, J. E. 1991, Nature, 354, 210

Hernquist, L. 1992, ApJ, 400, 460

Kennicutt, R. C., Schweizer, F., \& Barnes, J. E. 1998, 'Galaxies: Interactions and Induced Star Formation' (Berlin: Springer)

Knapen, J. H., Shlosman, I., \& Peletier, R. F. 2000, ApJ, 529, 93

Kormendy, J., \& Richstone, D. 1995, ARA\&A, 33, 581

Kormendy, J., et al. 1996, ApJ, 459, L57

Krabbe, A., et al. 1995, ApJ, 447, L95

Kuijken, K. 1995, in 'Barred Galaxies', Proc. IAU Symp. 157, Buta, R., Crocker, D. A., \& Elmegreen, B. G., eds., p. 504 (San Francisco: ASP)

Lauer, T. R., et al. 1995, AJ, 110, 2622

Lauer, T. R., et al. 1998, AJ, 116, 2263

Magorrian, J., et al. 1998, AJ, 115, 2285

Mehlert, D., Saglia, R. P., Bender, R., \& Wegner, G. 1998, A\&A, 332, 33

Merritt, D., \& Sellwood, J. A. 1994, ApJ, 425, 551

Mihos, J. C., \& Hernquist, L. 1994, ApJL, 437, L47

Mihos, J. C., \& Hernquist, L. 1996, ApJ, 464, 641

Najarro, F., Krabbe, A., Genzel, R., Lutz, D., et al. 1997, A\&A, 325, 700

Norman, C. A., Sellwood, J. A., \& Hasan, H. 1996, ApJ, 462, 114

Quinlan, G. D., \& Hernquist, L. 1997, New Astronomy, 2, 533

Raha, A., Sellwood, J. A., James, R., \& Kahn, F.D. 1991, Nature, 352, 411

Stanford, S. A., \& Bushouse, H. A. 1991, ApJ, 371, 92

van den Bosch, F., Jaffe, W., \& van der Marel, R. P., 1998, MNRAS, 293, 343

van Dokkum, P. G., Franx, M. 1995, AJ, 110, 2027

van der Marel R. P., van den Bosch, F. C. 1998, AJ, 116, 2220

van der Marel, R. P. 1999, AJ, 117, 744

van der Marel, R. P., \& Zurek D. 2000, in 'Dynamics of Galaxies: from the Early Universe to the Present', Combes F., Mamon G. A., Charmandaris V., eds., p. 504 (San Francisco: ASP 197)

van der Marel, R. P., Gerssen, J., Mihos, C., Axon, D., Hernquist, L., \& Barnes, J. 2001, in preparation

Verdoes Kleijn, G. A., Baum, S. A., de Zeeuw, P. T., \& O’Dea, C. P. 1999, AJ, 118, 2592

Vignati, P., et al. 1999, A\&A, 349, L57

Whitmore, B. C., Zhang, Q., Leitherer, C., Fall, S. M., Schweizer, F., Miller, B. W. 1999, AJ, 118, 1551

Wyse, R. F. G., Gilmore, G., \& Franx, M. 1997, ARAA, 35, 637

Young, P. 1980, ApJ, 242, 1232 